\title{
Efficient G-band Digital Communications Using Continuous Phase Modulation
}

\author{
J. X. Qiu, C.R. Dietlein, T.G. Ivanov, D.A. Wikner, A.S. Hedden, E.A. Viveiros, and H.A. Hung \\ U.S. Army Research Laboratory, Adelphi, MD, 20783, USA
}

\begin{abstract}
The use of continuous phase modulation (CPM) for digital communications at G-band $(216 \mathrm{GHz})$ has been investigated and demonstrated. The unique combination of constant envelope and multi-level symbol-maps of CPM offer the advantages of simultaneous high power-efficiency and spectralefficiency. A transmitter/receiver communication link consisting of an I/Q modulator followed by a multiplier is used to generate 16-level CPM waveforms at $216 \mathrm{GHz}$. Waveforms with minimal distortion at the full saturated power of the transmitter were demonstrated without output power backoff. The system architecture provides an efficient technique for generating high power digital waveform, thus extending the link distance. The technique is ideal for upper millimeter-wave and terahertz communications where output power is limited.
\end{abstract}

Index Terms - Terahertz, millimeter-wave, digital communications, continuous phase modulation, linearity.

\section{INTRODUCTION}

Systems operating at upper millimeter-wave and terahertz (MMW/THz) frequencies have a number of unique characteristics that can offer specific benefits for communication applications [1][2]. Their available wide bandwidth and high carrier frequency have the potential to support broadband data transmission in small operation platforms. They can also provide secure communication links due to their high directionality for a given compact physical aperture.

A number of experiments have reported $\mathrm{MMW} / \mathrm{THz}$ communication links using complex digital waveforms such as quadrature amplitude modulation (QAM) [3][4]. However, the available transmitter power was low, resulting in short range. The low transmission power is due to the large peak-toaverage-power-ratio (PAPR) of QAM waveforms, and additionally, the hardware limitations above $100 \mathrm{GHz}$, such as the lack of linear high power amplifiers and the low conversion efficiency of subharmonic mixers. These limitations together with high free-space path loss and atmospheric absorption have restricted the use of $\mathrm{MMW} / \mathrm{THz}$ for communications.

Continuous phase modulation (CPM) is a class of constantenvelope waveforms that is jointly power and bandwidth efficient [5]. It has been used in applications with high-loss nonlinear channels such as satellite Communications [6] and cellular wireless communications (GSM). It is also being considered for $60 \mathrm{GHz}$ wireless communications [7].
In this paper, the use of multi-level CPM for $\mathrm{MMW} / \mathrm{THz}$ communications is presented. A system consisting of an I/Q modulator followed by a multiplier chain is used to demonstrate the generation of CPM waveforms at $216 \mathrm{GHz}$. Distortion-free waveforms at the full output power $(5 \mathrm{dBm})$ of the multiplier were generated, transmitted and received without output power backoff. To the best of the authors' knowledge, this represents the highest power for multi-level digitally modulated waveform at this frequency range, which is a result of highly efficient use of the available transmit power.

\section{THEORY OF CPM}

A CPM waveform is described by[5]

$$
s(t)=A \cos \left[2 \pi f_{c} t+\phi(t, \vec{a})\right]
$$

where the signal amplitude $A$ is constant in time, and $f_{\mathrm{c}}$ is the carrier frequency. The transmitted information is contained in the phase

$$
\phi(t, \vec{a})=2 \pi h \sum_{k=-\infty}^{\infty} a_{k} q(t-k T)
$$

where $h$ is modulation index, and $\vec{a}=\left\{a_{k}\right\}$ is the transmitted $M$-ary symbol sequence. The $M$-ary symbols $a_{k}$ take the values of $\pm 1, \pm 3, \ldots, \pm(M-1) ; M$ is usually a power of $2 . T$ is the symbol period. The phase function is given by

$$
q(t)=\int_{-\infty}^{t} g(\tau) d \tau
$$

The function $g(t)$ is the frequency shape pulse and is usually normalized such that

$$
\int_{-\infty}^{\infty} g(t) d t=1 / 2
$$

This makes the maximum phase change of the signal to be $(M-1) h \pi$ over the period of $g(t)$. By choosing different pulses $g(t)$ and varying the modulation index $h$ and $M$, a variety of CPM schemes can be obtained.

If a CPM waveform as defined by (1) is passed through a frequency multiplier, the output waveform is given by

$$
s(t)=A \cos \left[2 \pi n f_{c} t+n \phi(t, \vec{a})\right]
$$

where $n$ is the multiplication factor. The waveform in (5) remains a CPM waveform with multiplications in both carrier frequency and modulation index. 


\section{EXPERIMENTAL SETUP}

The block diagram of the experimental setup to demonstrate the generation and transmission of CPM at $216 \mathrm{GHz}$ is shown in Fig. 1. It includes a transmitter and receiver. The key components of the transmitter consist of a Tektronix AWG5014 arbitrary waveform generator (AWG) for generating the digital baseband waveforms, a direct inphase/quadrature (I/Q) modulator for up-converting the baseband waveforms to an intermediate frequency (IF) of 13.5 GHz, and a Virginia Diodes Inc. (VDI) multiplier chain (x16) to multiply the IF to $216 \mathrm{GHz}$. The local oscillator (LO) for the I/Q modulator is provided by an Agilent E8257D low phase-noise frequency synthesizer.

The receiver includes a VDI subharmonic mixer (SHM) driven by a LO which is up-converted from $12.08 \mathrm{GHz}$ by another VDI multiplier chain (x9). The $12.08 \mathrm{GHz}$ signal is provided by an Agilent E8257D low phase-noise frequency synthesizer. The $1.44 \mathrm{GHz}$ IF from the SHM is captured by an Agilent E4448A $50 \mathrm{GHz}$ PSA spectrum analyzer with a builtin down-converter and an $80-\mathrm{MHz}$ bandwidth digitizer. The Agilent 89600 VSA software running on an external computer is used to download the captured waveforms from the PSA, and performs demodulation and analysis on the waveforms. If the IF waveform bandwidth is greater than $80 \mathrm{MHz}$, the waveform is directly captured by an Agilent DSA90404A digital oscilloscope with $4 \mathrm{GHz}$ bandwidth. The VSA software is then used to acquire, demodulate and analyze the waveform.

A set of specific MATLAB programs running with the Agilent VSA software has been developed to control both the transmitter and the receiver. These programs were responsible for generating digital waveforms, transferring waveforms from the VSA software into the MATLAB environment for processing as well as system calibration.

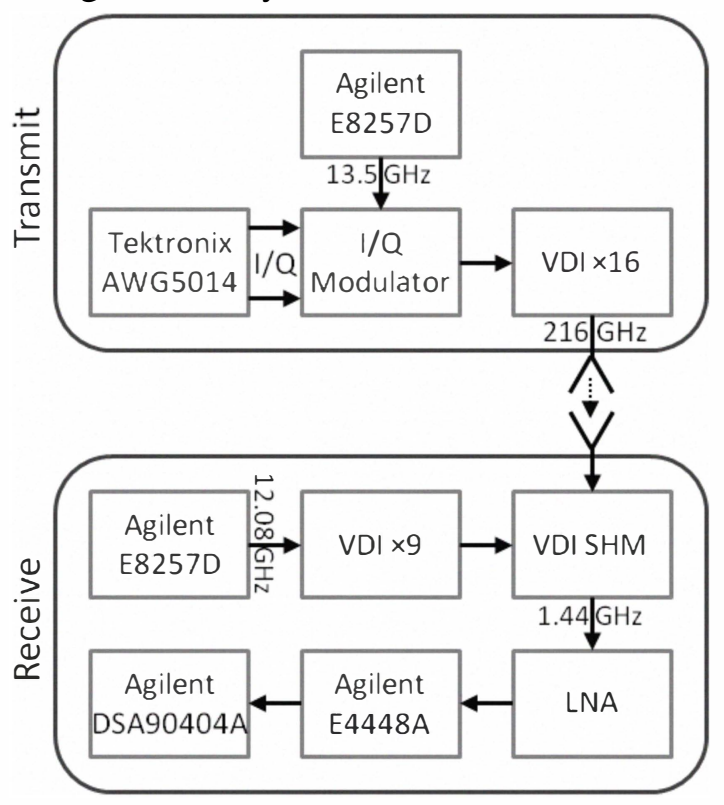

Fig. 1. Block diagram of the $216 \mathrm{GHz}$ communication link.
The output of the transmitter multiplier chain and the input of the receiver SHM are connected to two horn antennas with a gain at $216 \mathrm{GHz}$ of $21.5 \mathrm{~dB}$ each. The two horns are separated by $0.4 \mathrm{~m}$.

\section{COMM-LINK DEMONSTRATION AT $216 \mathrm{GHz}$}

The AWG was programmed to generate the baseband waveforms to produce constant-envelope CPM waveform at the output of the I/Q modulator at $13.5 \mathrm{GHz}$. Waveforms with different modulation indices, levels and speeds were generated. In Fig. 2, the envelope and spectrum of a 16-level CPM waveform are shown. The symbol rate $(1 / \mathrm{T})$ of the waveform is $10 \mathrm{Msymbols} / \mathrm{s}$ which corresponds to an aggregate bit rate of $40 \mathrm{Mbits} / \mathrm{s}$. The frequency shape pulse $g(t)$ is a Gaussian pulse with a $3-\mathrm{dB}$ bandwidth (B) and symbol period (T) product of $\mathrm{BT}=0.5$. The modulation index $h$ is 0.025 . Fig. 2(a) shows the constant-envelope characteristic of the waveform. In Fig. 3, the demodulated eye diagram of the waveform at $13.5 \mathrm{GHz}$ is shown. The waveform was demodulated as frequency shift keying (FSK). The quality of the waveform is measured by FSK error defined as the RMS deviation between the measured demodulated frequencies and the reference symbol frequencies. FSK error is similar to error-vector-magnitude (EVM) but defined in demodulated
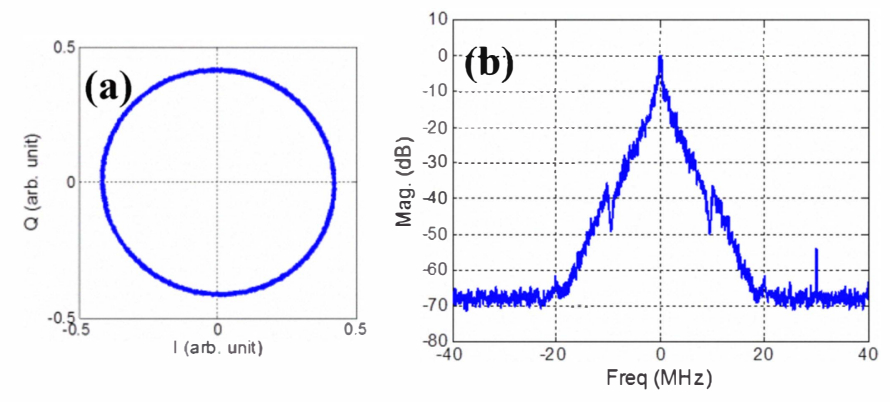

Fig. 2. Transmitter signal envelop (a), and power spectral density (b) of a 16-level CPM waveform at $13.5 \mathrm{GHz}$ with Gaussian shape pulse. $(1 / \mathrm{T}=10 \mathrm{MHz}, \mathrm{BT}=0.5, \mathrm{~h}=0.025)$

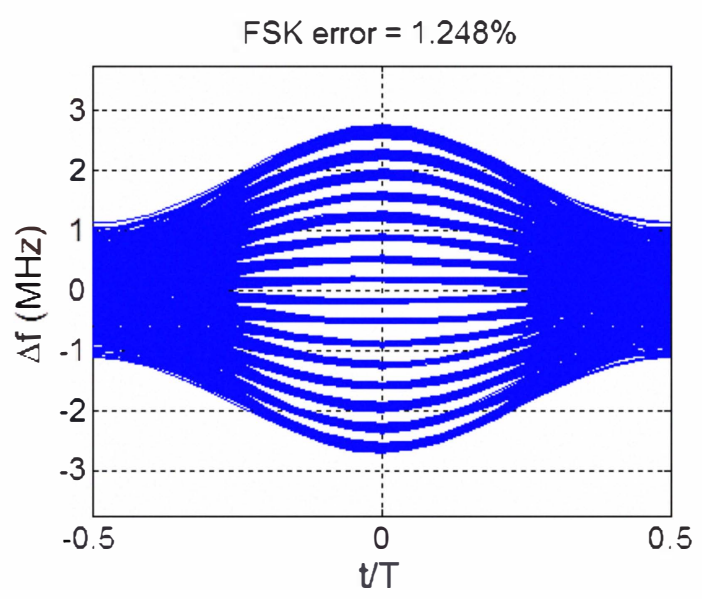

Fig. 3. Transmitter signal eye diagram for the CPM waveform at $13.5 \mathrm{GHz}$. 
frequency space instead of the I/Q plane and can be used to estimate bit-error-rate. A low FSK error of $1.25 \%$ was measured at the output of the I/Q modulator.

Fig. 4 shows the envelope and spectrum of the CPM waveform in Fig. 2 after it was multiplied in frequency to 216 $\mathrm{GHz}$, transmitted over free space, down-converted to $1.44 \mathrm{GHz}$ by the receiver. The envelope of waveform remains constant while its spectrum expands due to frequency multiplication. The spectral growth is also verified in the eye diagram in Fig. 5. The maximum FSK frequency deviations at $216 \mathrm{GHz}$ and $13.5 \mathrm{GHz}$ are approximately $41.9 \mathrm{MHz}$ and $2.6 \mathrm{MHz}$, respectively. The FSK error at $216 \mathrm{GHz}$ is about $1.26 \%$. The power at the output of the transmitter was measured with an Erickson PM4 power meter. The measured output power was approximately $5 \mathrm{dBm}$, which was also the maximum output power of the multiplier with $\mathrm{CW}$ input and independent of modulation index and level.

To study the link power margin, a variable attenuator was inserted between the multiplier output and the transmit antenna horn. With greater than $40 \mathrm{~dB}$ additional attenuation, the received eye diagram remained open and the FSK error was only increased to $1.8 \%$. Further increase in the path attenuation eventually closed the eye diagram. Assuming a current link margin of $40 \mathrm{~dB}$, the maximum range of the current setup is about $40 \mathrm{~m}$. If high-gain antennas are used, longer range can be achieved. For example, the maximum range is $10 \mathrm{~km}$ if both the transmitter and the receiver antennas have $60 \mathrm{~dB}$ gain. This calculation takes into account free space loss as well as atmospheric loss including water vapor and oxygen [8]. A long-range experiment incorporating solid-state power amplifiers at $216 \mathrm{GHz}$ in the link is on-going.

\section{CONCLUSION}

For the first time, an efficient communication link architecture based on multi-level CPM waveforms has been demonstrated at greater than $100 \mathrm{GHz}$. The constant-envelope characteristic of CPM enables generation of high-power, high purity spectrum without output power backoff, thus offering long range $\mathrm{MMW} / \mathrm{THz}$ digital communications, with maximum available transmit power, and minimal distortion in the received signal. When high power $\mathrm{MMW} / \mathrm{THz}$ amplifiers are incorporated, the constant-envelope characteristic of CPM will ensure the amplifiers maintain high output power and high efficiency.

\section{ACKNOWLEDGEMENT}

The authors would like to thank Dr. K. Ikossi of DTRA for supports, Drs. B. Danly and H. Newman of NRL for useful discussions.
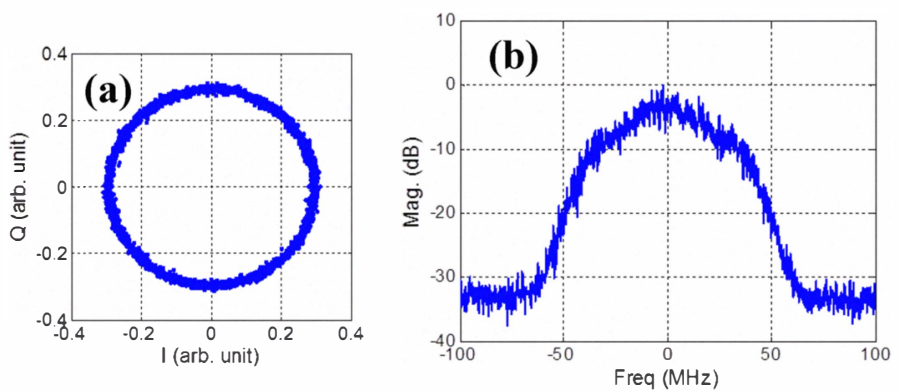

Fig. 4.Receiver signal envelope (a), and power spectral density (b) of the CPM waveform in Fig. 2 after it is down converted from $216 \mathrm{GHz}$.

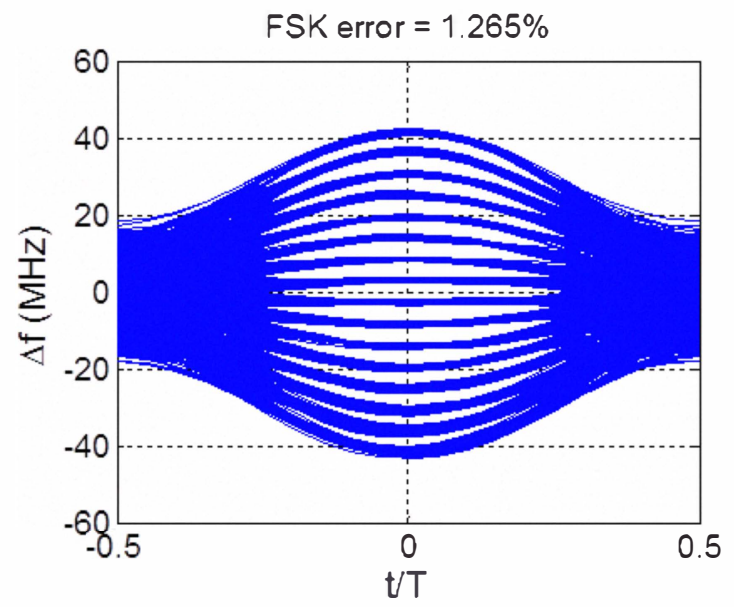

Fig. 5. Receiver eye diagram for the CPM waveform in Fig. 4.

\section{REFERENCES}

[1] J. Federici and L. Moeller, "Review of terahertz and subterahertz wireless communications," J. Appl. Phy., vol. 19, pp. $1111011-22,2010$

[2] T. Kleine-Ostmann and T. Nagatsuma, "A review on terahertz communications research," J. of Infrared Millim. Terahertz Waves, vol. 32, pp. 143-171, 2011.

[3] C. Jastrow, et al., "Wireless digital data transmission at 300 GHz," Elect. Lett, vol. 46, pp. 661-662, April 2010.

[4] I. Kallfass, et al., "All Active MMIC-Based Wireless Communication at $220 \mathrm{GHz}$," IEEE Trans. Terahertz Sci. Tech., vol. 1, no. 2, pp. 477-487, November 2011.

[5] C.-E. Sundberg, "Continuous phase modulation: a class of jointly power and bandwidth efficient digital modulation schemes with constant amplitude," IEEE Comm. Mag., vol. 24, no. 4, pp. 25-38, April 1986.

[6] J.H. Yuen, et al., "Modulation and coding for satellite and space communications," Proc. IEEE, vol. 78. No. 7, pp. 1250-1266, July 1990.

[7] C-C. Chong, et al., Editors, Millimeter-Wave Wireless Communication Systems: Theory and Applications, Hindawi Publishing, 2006.

[8] H.B. Wallace, "AEM" software (www.mmwconcepts.com). 